https://idp.uoc.edu

ARTÍCULO

\title{
Presentación del monográfico sobre la COVID-19
}

\author{
Ivan Serrano \\ Profesor de Derecho y Ciencias Políticas \\ Universitat Oberta de Catalunya
}

Fecha de publicación: marzo de 2021

Este número de IDP. Revista de Internet, Derecho y Política recoge las contribuciones surgidas a partir de la convocatoria especial que realizó la revista con motivo de la crisis de la COVID-19. El enorme e incierto impacto que esta situación está generando en nuestras sociedades ponía de manifiesto la importancia de analizar aquellos aspectos relacionados con las tecnologías de la información y la comunicación en los ámbitos propios de la revista como son el derecho, la administración pública, la política, la resolución de conflictos, la criminología, las relaciones internacionales o la ciudad.

Los artículos que presentamos en este número especial recogen ciertamente esta diversidad de aproximaciones, señalando también las múltiples implicaciones de la actual crisis en diversos ámbitos de nuestras sociedades. Unas implicaciones que con toda seguridad serán objeto de estudio ineludible para las ciencias sociales y donde este número de la revista quiere ser una contribución tanto para el análisis actual como para la agenda futura de investigación.

El primer artículo recogido en este número expone el trabajo de Francisca Ramón Fernández, bajo el título «La desconexión digital y docencia universitaria online en tiempos de pandemia por la COVID-19: una ilusión más que una realidad». La autora analiza el impacto en la actividad laboral que está teniendo la pandemia en el sector de la docencia universitaria. Señala el artículo cómo la situación actual está poniendo a prueba regulaciones anteriores en el ámbito de los derechos digitales, señalando algunas de sus limitaciones, como aquellas referentes a la conciliación entre vida laboral y familiar, debido al fenómeno de la hiperconexión, y cómo regulaciones posteriores en esta materia pueden o no ofrecer un marco regulador más adecuado. 
El artículo de Miguel Ángel Sevilla, «Las medidas de contención de la COVID-19 frente al derecho a la protección de datos personales sanitarios de la CDFUE», aborda la protección de datos en el ámbito de la gestión sanitaria, un aspecto relevante puesto que su buen uso es un factor importante para la elaboración de políticas públicas. Como señala el autor, algunas de estas mesuras pueden contravenir disposiciones respecto a la protección de datos personales, por lo que es necesario estudiar las implicaciones y las vías de conciliación entre la importancia de su uso -mediante por ejemplo técnicas de Big Data- para combatir la pandemia y la protección de derechos básicos como la privacidad.

En un ámbito diferente, el artículo «Crimen, cibercrimen y COVID-19: desplazamiento (acelerado) de oportunidades y adaptación situacional de ciberdelitos», de Fernando Miró-Llinares, aborda el impacto que este nuevo contexto tiene en la cibercriminalidad. A partir de una revisión de los trabajos existentes y de la evidencia original aportada por el propio estudio, los resultados sugieren que las prácticas de cibercrimen se han adaptado ciertamente a las nuevas oportunidades surgidas del actual contexto, donde se ha incrementado sustancialmente la digitalización en la práctica cotidiana de nuestras sociedades. A pesar de ello, el artículo señala como este proceso de digitalización no es novedoso en sí, de modo que cabe concebir la crisis de la COVID-19 como un acelerador más que como un factor causal novedoso.

En el artículo de Cristina Güerri, Marta Martí y Albert Pedrosa «Abriendo ventanas virtuales en los muros de la prisión. En torno a la digitalización penitenciaria a propósito de la COVID-19», se aborda un ámbito de investigación relevante -más aún en el contexto de la COVID-19-como son sus repercusiones en el ámbito de las prisiones. Los numerosos elementos que son objeto de preocupación en el ámbito penitenciario se ven amplificados por los riesgos derivados de las peculiaridades de la reclusión. Una de las mesuras aplicadas, suspender permisos y visitas, tiene unas derivadas importantes en relación con uno de los objetivos fundamentales de la política penitenciaria como es la reinserción social de los presos. El trabajo tiene como objetivo resaltar esta importancia -mayor aún dada la situación actual-y cómo la insuficiente digitalización de las prisiones no permite aprovechar el potencial que tendría para facilitar vías de comunicación entre las personas presas y sus allegados.

Por último, en relación con otro aspecto que se viene discutiendo durante los últimos meses, el artículo «COVID-19, alquiler turístico y políticas de cancelación: ¿Emergencia en tiempos de pandemia de la oculta(da) naturaleza de las plataformas digitales?», de Apol|lònia Martínez Nadal, incide en cómo la situación actual pone de manifiesto los límites de ciertos conceptos jurídicos tradicionales de aplicación en el ámbito del comercio electrónico. El análisis del pronunciamiento por parte del Tribunal de Justicia de la Unión Europea sobre la condición de Airbnb nos indica, según la autora, la necesidad de nuevas categorías jurídicas respecto a las plataformas digitales, lo que el actual contexto ha evidenciado aún más dada la posición de fuerza que tienen dichas plataformas, que son mucho más que meras intermediarias neutrales entre terceros. 
En definitiva, los artículos recogidos en el presente número muestran, como se señalaba al principio, no solo la relevancia que tiene la investigación desde los ámbitos propios de la revista en el contexto de la actual pandemia de la COVID-19, sino la importancia de señalar aspectos que, más allá del día a día de la actualidad ligados a sus efectos inmediatos, tendrán fuertes implicaciones que se consolidarán a largo plazo y que generarán una agenda de investigación necesaria en relación con los retos a los que se enfrentan nuestras sociedades.

\section{Cita recomendada}

SERRANO, Ivan (2021). «Presentación del monográfico sobre la COVID-19». IDP. Revista de Internet, Derecho y Política, núm. 32 (marzo). UOC [Fecha de consulta: dd/mm/aa] http://dx.doi.org/10.7238/idp.v0i32.381596

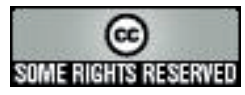

Los textos publicados en esta revista están -si no se indica lo contrario- bajo una licencia Reconocimiento-Sin obras derivadas 3.0 España de Creative Commons. Puede copiarlos, distribuirlos y comunicarlos públicamente siempre que cite su autor y la revista y la institución que Ios publica (IDP. Revista de Internet, Derecho y Política; UOC); no haga con ellos obras derivadas. La licencia completa se puede consultar en: http://creativecommons.org/ licenses/by-nd/3.0/es/deed.es.

\section{Sobre el autor}

Ivan Serrano

Profesor de Derecho y Ciencias Políticas

Universitat Oberta de Catalunya 